\title{
Score Selection Techniques for Fingerprint Multi-modal Biometric Authentication
}

\author{
Giorgio Giacinto, Fabio Roli, and Roberto Tronci \\ Department of Electric and Electronic Engineering, \\ University of Cagliari, Piazza D'Armi, I-09123 Cagliari, Italy \\ \{giacinto, roli, roberto.tronci\}@diee.unica.it
}

\begin{abstract}
Fingerprints are one of the most used biometrics for automatic personal authentication. Unfortunately, it is often difficult to design fingerprint matchers exhibiting the performances required in real applications. To meet the application requirements, fusion techniques based on multiple matching algorithms, multiple fingerprints, and multiple impressions of the same fingerprint, have been investigated. However, no previous work has investigated selection strategies for biometrics. In this paper, a score selection strategy for fingerprint multi-modal authentication is proposed. For each authentication task, only one score is dynamically selected so that the genuine and the impostor users' scores distributions are mainly separated. Score selection is performed by first estimating the likelihood that the input pattern is an impostor or a genuine user. Then, the min score is selected in case of an impostor, while the max score is selected in case of a genuine user. Reported results show that the proposed selection strategy can provide better performances than those of commonly used fusion rules.
\end{abstract}

\section{Introduction}

Multimodal biometric systems have been proposed to increase the accuracy of authentication systems 4. According to Prabhakar and Jain 10 multimodal biometric systems can be subdivided into five different scenarios: multiple biometrics [1 5] 11, multiple acquisitions of the same biometry with the same sensor 6], multiple representations, and matching algorithms for the same biometry [8] [10, multiple units of the same biometry (for example different fingers) [6], multiple sensors for the same biometry 9. In this paper we use fingerprints as biometrics.

The vast majority of multimodal systems are based on fusion strategies at the score level, so that the score of different matchers are combined to attain a "new" score. Typical fusion rules adopted for multimodal system are the min, max, median, mean, as well as trainable rules such as neural networks [9]. However, the output of different matchers could be exploited not only by fusion rules, but also by dynamic selection mechanisms. In the pattern recognition field, selection mechanisms have been proposed to select, for each input pattern, the classifier that provides the correct output [7. This formulation of the selection problem 
requires that each classifier outputs a class label for each input pattern. An example of selection mechanism is the algorithm for Dynamic Classifier Selection (DCS) that estimates the competence of each classifier by computing the local accuracy in a neighborhood of the test pattern [2] [12].

It is easy to see that the use of DCS mechanisms for multimodal biometric systems is not straightforward because: (a) it is hard to define a "local neighborhood" of the input pattern where accuracy should be computed; (b) the computation of the accuracy requires that for each matcher an acceptance threshold is set. It is worth pointing out that the vast majority of multimodal systems employs fusion rules at the score-level to avoid setting acceptance thresholds, as it is difficult to relate the choice of the thresholds of individual matchers to the performances of fusion rules at the decision level. On the other hand, fusion rules at the score-level aim at producing distributions of scores allowing for better discrimination between legitimate users and impostors.

In this paper we formulate a selection mechanisms for multimodal systems aimed at separating the distributions of scores of genuine and the impostor users. First, the available scores are used to predict the most likely state of nature of the input pattern, i.e. impostor or genuine. Then, the maximum score is chosen if the pattern is likely to be a genuine, while the minimum score is chosen if the pattern is likely to be an impostor.

The rest of the paper is organized as follows: Section 2 proposes an ideal selector for a multimodal systems. In Section 3 an algorithm based on the ideal selector is proposed. Experimental results on a fingerprint dataset are presented in Section 4 Section 5 concludes the paper.

\section{Dynamic Score Selection for Multimodal Systems}

In the field Multiple Classifier Systems, the oracle has been defined as the ideal selector which always selects, for each input pattern, the classifier that provides the correct label, if any. Accordingly, some algorithms have been proposed in the literature 3 .

In a recent paper this definition of the oracle has been applied to multimodal systems where for each matcher an acceptance threshold has been set in advance 9. By setting an acceptance threshold, each matcher can be considered as a classifier whose output is an accept/reject decision. As a consequence, an oracle has been defined as the ideal selector which selects the matcher, if any, that correctly authenticated or rejected the input fingerprint in the case of a genuine or impostor user, respectively.

In this paper we propose a different formulation of the selection problem, that does not require setting acceptance thresholds for the available matchers. Thus, each matcher is not considered as a classifier, and selection is performed at the score level, so that, for each input pattern, only one of the available scores is selected. Such a selection mechanism should produce a distribution of impostor and genuine scores separated as much as possible. To attain this result, such an ideal selector should choose, for each pattern, the largest score in case of patterns 

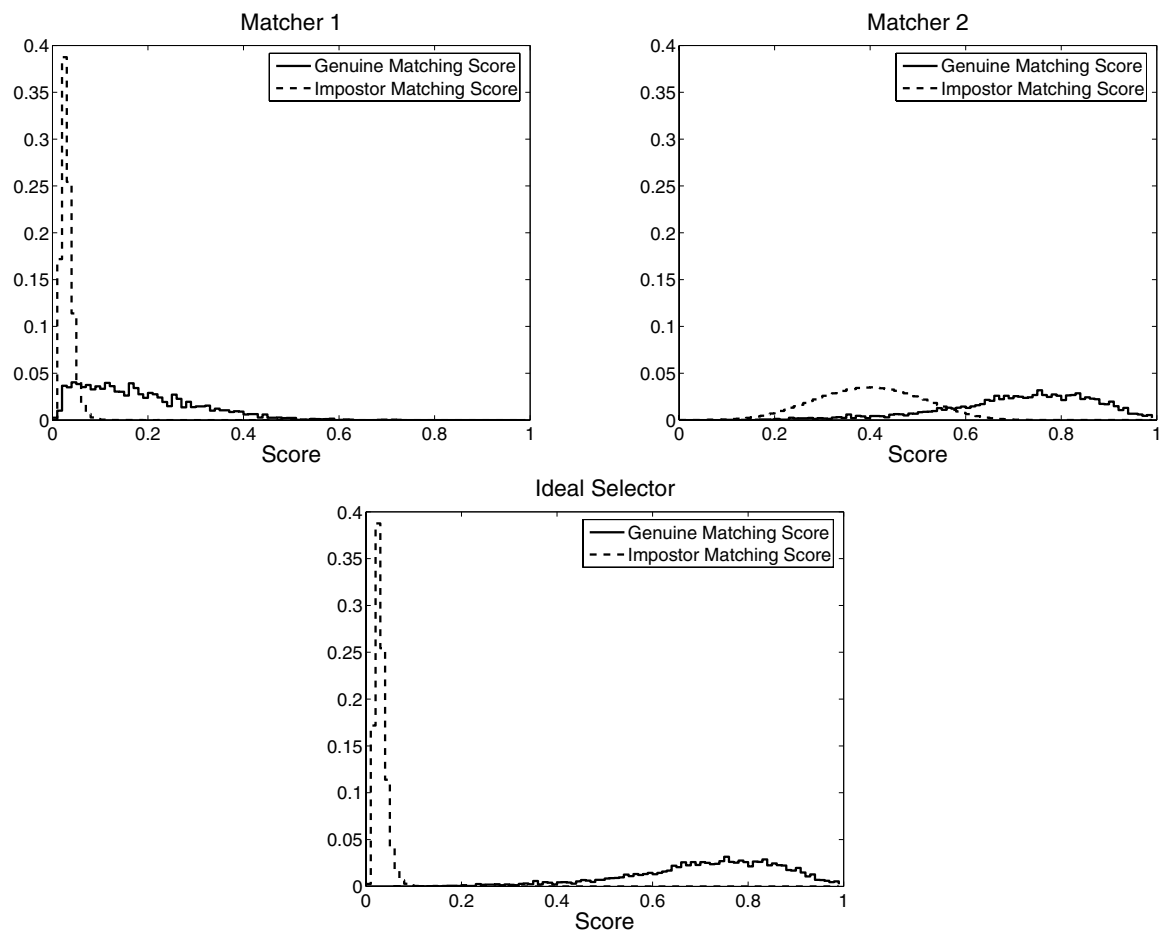

Fig. 1. An example of ideal selector with two matchers

belonging to genuine users, and the smallest score in case of patterns belonging to impostors. In other words, the ideal selector can be viewed as a switch between the Max and the Min fusion rules. Figure 1 shows an example of the selection rule implemented by the proposed ideal selector. It is easy to see that the use of the Max rule for genuine users produces a genuine score distribution shifted toward large score values, while the Min rule in case of impostors produces an impostor score distribution shifted toward small score values. In addition, with respect to the score distributions produced by the available sensors, the two distributions produced by the ideal selector exhibit a larger distance between the means, and a smaller variance.

In order to show why score selection should work, let us recall the two errors commonly used to evaluate authentication systems for a given acceptance threshold $s^{*}$, i.e. the False Acceptance Rate (FAR)

$$
\begin{aligned}
F A R\left(s^{*}\right) & =P\left(s>s^{*} \mid \text { impostor }\right)=\int_{s^{*}}^{1} p(s \mid \text { impostor }) \mathrm{d} s= \\
& =1-\int_{0}^{s^{*}} p(s \mid \text { impostor }) \mathrm{d} s
\end{aligned}
$$

and the False Rejection Rate (FRR)

$$
F R R\left(s^{*}\right)=P\left(s<s^{*} \mid \text { genuine }\right)=\int_{0}^{s^{*}} p(s \mid \text { genuine }) \mathrm{d} s
$$


It can be shown that for a given value of FAR the ideal selector exhibits a FRR smaller than that of any of the available matcher, and that for a given value of FRR the ideal selector exhibits a FAR smaller than that of any of the available matcher. According to the definition of ideal selector, it can be easily seen that for a given value of the threshold $s^{*}$

$$
\begin{array}{ll}
F A R_{\text {ideal selector }}\left(s^{*}\right) \leq F A R_{j}\left(s^{*}\right) & j=1, \ldots N \\
F R R_{\text {ideal selector }}\left(s^{*}\right) \leq F R R_{j}\left(s^{*}\right) & j=1, \ldots N
\end{array}
$$

where $\mathrm{N}$ is the number of available matcher.

Thus it follows that, if the value of FAR is fixed, different thresholds have to be set, the threshold of the matchers being always larger than or equal to that of the ideal selector. As a consequence, the FRR of the matchers are always larger than the FRR of the ideal selector. The same reasoning can be used to show that for a fixed value of FRR, the FAR of the matchers is always larger than or equal to the FAR of the ideal selector.

\section{A Score Selection Algorithm}

Let us assume that a set of $N$ matchers $M=\left\{M_{1}, M_{2} \ldots M_{N}\right\}$ is available. Each matcher $M_{j}$ outputs a score $s_{j}$ for each pattern $x$. Moreover, let us use the Gaussian model for the genuine user and impostor distributions.

An algorithm for selection based on the ideal selector described in Section 2 should be made up of two steps:

1. Estimation of the more likely state of nature $\omega$ of the pattern $x$, where $\omega \in\{$ genuine, impostor $\}$

2. Selection of the score $s_{\text {sel }}$ based on the estimated state of nature $\omega$ as

$$
s_{\text {sel }}= \begin{cases}\max _{j}\left(s_{j}\right) & \text { if } \quad \omega=\text { genuine } \\ \min _{j}\left(s_{j}\right) & \text { if } \quad \omega=\text { impostor }\end{cases}
$$

In order to estimate the state of nature of pattern $x$, for each matcher $M_{j}$ $(j=1 \ldots N)$, a measure of the expected error in classifying the pattern as being a genuine or an impostor is computed (Section 3.1). In the rest of the paper we will refer to this algorithm as Dynamic Score Selection (DSS).

\subsection{Minimum Expected Error}

Let us assume that for any value of $s^{*}$, the following relation holds

$$
\int_{0}^{s^{*}} p(s \mid \text { impostor }) \mathrm{d} s>\int_{0}^{s^{*}} p(s \mid \text { genuine }) \mathrm{d} s
$$

i.e., in the range $\left[0, s^{*}\right]$ impostors outnumber genuine users. This is usually true for a wide range of values of $s^{*}$. 
In order to compute the expected error in assigning a pattern to one of the classes "impostor" or "genuine" given the output score $s_{j}$ of the $j$ th matcher, let us set the acceptance threshold to $s_{j}$ and compute the difference

$$
D_{j}=\left|F R R\left(s_{j}\right)-F A R\left(s_{j}\right)\right|
$$

By substituting Equations (11) and (2) in (41),

$$
\mid \int_{0}^{s^{*}} p(s \mid \text { genuine }) \mathrm{d} s-1+\int_{0}^{s^{*}} p(s \mid \text { impostor }) \mathrm{d} s \mid
$$

If $F R R\left(s_{j}\right)>F A R\left(s_{j}\right)$, then $\int_{0}^{s^{*}} p(s \mid$ genuine $) \mathrm{d} s-1+\int_{0}^{s^{*}} p(s \mid$ impostor $) \mathrm{d} s=$ $D_{j}>0$. According to the assumption in Equation (3) we have

$$
\begin{aligned}
& \int_{0}^{s^{*}} p(s \mid \text { impostor }) \mathrm{d} s> \int_{0}^{s^{*}} p(s \mid \text { genuine }) \mathrm{d} s=D_{j}+1-\int_{0}^{s^{*}} p(s \mid \text { impostor }) \mathrm{d} s \\
& \int_{0}^{s^{*}} p(s \mid \text { impostor }) \mathrm{d} s>\frac{D_{j}+1}{2} \\
& F A R\left(s_{j}\right)=1-\int_{0}^{s^{*}} p(s \mid \text { impostor }) \mathrm{d} s<\frac{1-D_{j}}{2}
\end{aligned}
$$

Thus it follows that by accepting the input pattern as a genuine user an error smaller than $\left(1-D_{j}\right) / 2$ is expected. As a consequence the input pattern is likely to be a genuine user.

Analogously, if $F A R\left(s_{j}\right)>F R R\left(s_{j}\right)$, thus it follows that $F R R\left(s_{j}\right)<(1-$ $\left.D_{j}\right) / 2$, and the input pattern is likely to be an impostor. The highest the value of $D_{j}$ computed according to Equation (4), the most likely the decision, as it leads to the minimum expected error. Summing up, the state of nature $\omega$ for pattern $x$ can be estimated as follows:

1. let us compute, for each matcher $M_{j}$, the value of $D_{j}$

2. let $k=\operatorname{argmax}_{j}\left(D_{j}\right)$

3 . then

$$
\omega=\left\{\begin{array}{lll}
\text { genuine } & \text { if } & F R R\left(s_{j}\right)>F A R\left(s_{j}\right) \\
\text { impostor } & \text { if } & F A R\left(s_{j}\right)>F R R\left(s_{j}\right)
\end{array}\right.
$$

The difference in Equation (4) can be estimated by assuming Gaussian distributions for the score of genuine users and impostors. Let $\mu_{G}\left(\mu_{I}\right)$ and $\sigma_{G}\left(\sigma_{I}\right)$ be the mean and the standard deviation of genuine (impostor) distribution estimated from the training set. Let us also consider the first-order approximation of the integral used to compute the errors

$$
P\left(s<s^{*}\right)=\frac{1}{2}\left(1+\frac{2}{\sqrt{\pi}} \frac{s^{*}-\mu}{\sqrt{2} \sigma}\right)
$$

Thus, substituting Equation (6) in Equation (4) we obtain:

$$
\begin{gathered}
D_{j}=\left|F R R\left(s_{j}\right)-F A R\left(s_{j}\right)\right|= \\
=\mid P\left(s<s_{j} \mid \text { genuine }\right)-P\left(s>s_{j} \mid \text { impostor }\right) \mid=
\end{gathered}
$$




$$
\begin{gathered}
=\mid P\left(s<s_{j} \mid \text { genuine }\right)-\left[1-P\left(s<s_{j} \mid \text { impostor }\right)\right] \mid= \\
=\left|\frac{1}{2}\left(1+\frac{2}{\sqrt{\pi}} \frac{s_{j}-\mu_{G}}{\sqrt{2} \sigma_{G}}\right)-1+\frac{1}{2}\left(1+\frac{2}{\sqrt{\pi}} \frac{s_{j}-\mu_{I}}{\sqrt{2} \sigma_{I}}\right)\right|= \\
=\frac{1}{\sqrt{2 \pi}}\left|\frac{s_{j}-\mu_{G}}{\sigma_{G}}+\frac{s_{j}-\mu_{I}}{\sigma_{I}}\right|
\end{gathered}
$$

\subsection{Dynamic Score Selection with Selection Threshold}

It is easy to see that if the value of $D_{j}$ are close to zero, i.e. the scores are close to the Equal Error Rate (EER) point, then the above estimation of the most likely state of nature is not reliable. On the other hand, it could be a safe decision to consider the input pattern as an impostor, and produce the output score using the Min rule. To this end, if the maximum value of $D_{j}$ is smaller than some predefined threshold, than the Min rule is used.

\section{Experimental Results}

We tested the dynamic score selection technique on a fingerprint multisensor database that has been developed at our department [9. This database is divided into two parts, each one containing 1200 fingerprints related to an optical sensor (Biometrika FX2000), and a capacitive sensor (Precise Biometrics MC100), respectively. These fingerprints have been taken from 20 volunteers. For each volunteer, ten impressions of three different fingers of both hands were acquired. We performed two sets of experiments, namely multi-sensor and multi-algorithmic. Experiment 1 was performed using the "String" algorithm as minutiae matcher for both the available sensors. Experiment 2 was performed using the "String"

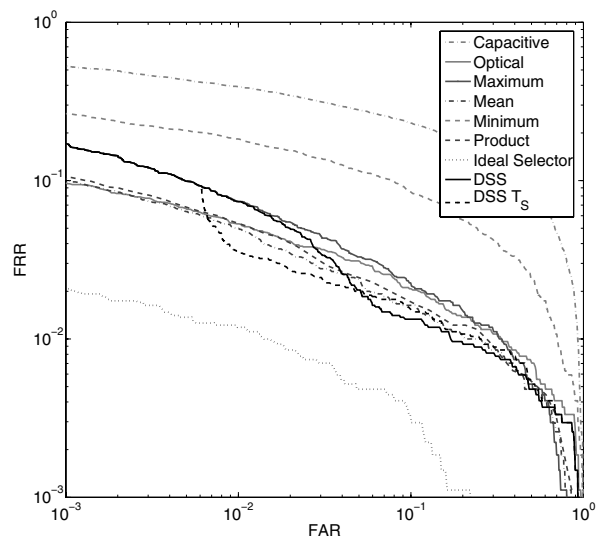

(a)

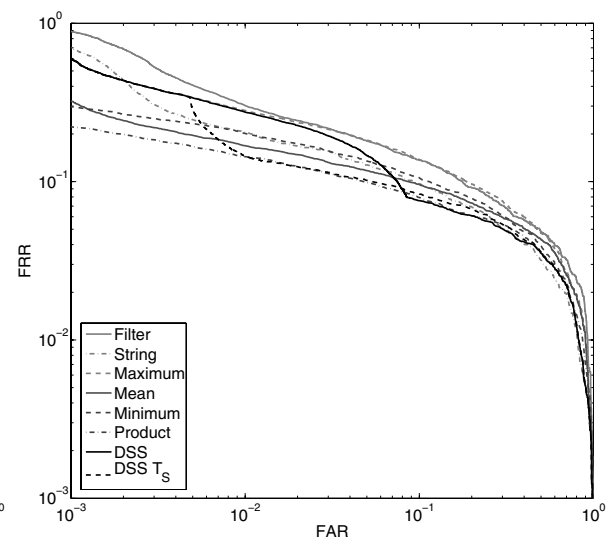

(b)

Fig. 2. R.O.C. curves for Experiment 1 (a) and Experiment 2 (b) 
Table 1. Error rates of individual and combined systems in Experiment 1

\begin{tabular}{cccc}
\hline Method & EER & 1\% FAR & 1\% FRR \\
\hline Capacitive & $18.65 \%$ & $39.15 \%$ & $92.67 \%$ \\
Optical & $3.52 \%$ & $5.33 \%$ & $33.44 \%$ \\
Maximum & $3.88 \%$ & $7.37 \%$ & $33.66 \%$ \\
Mean & $2.86 \%$ & $5.00 \%$ & $25.43 \%$ \\
Minimum & $9.14 \%$ & $18.33 \%$ & $71.03 \%$ \\
Product & $3.03 \%$ & $5.15 \%$ & $28.44 \%$ \\
Ideal Selector & $1.13 \%$ & $1.19 \%$ & $1.52 \%$ \\
$D S S$ & $3.26 \%$ & $7.37 \%$ & $17.01 \%$ \\
$D S S T_{S}$ & $2.57 \%$ & $3.52 \%$ & $25.41 \%$ \\
\hline
\end{tabular}

Table 2. Error rates of individual and combined systems in Experiment 2

\begin{tabular}{cccc}
\hline Method & EER & 1\% FAR & 1\% FRR \\
\hline Filter & $12.45 \%$ & $28.11 \%$ & $90.45 \%$ \\
String & $10.31 \%$ & $20.00 \%$ & $82.75 \%$ \\
Maximum & $12.45 \%$ & $28.15 \%$ & $90.47 \%$ \\
Mean & $9.67 \%$ & $16.87 \%$ & $89.94 \%$ \\
Minimum & $10.31 \%$ & $20.08 \%$ & $82.56 \%$ \\
Product & $8.44 \%$ & $14.36 \%$ & $86.45 \%$ \\
Ideal Selector & $0.04 \%$ & $0.02 \%$ & $0.00 \%$ \\
DSS & $8.31 \%$ & $27.45 \%$ & $82.04 \%$ \\
DSS TS & $8.67 \%$ & $14.53 \%$ & $82.04 \%$ \\
\hline
\end{tabular}

and "Filter" algorithms as minutiae matcher, and the capacitive sensor. The proposed DSS algorithm has been compared to four "fixed" fusion rules (Maximum, Mean, Minimum and Product). Performances were assessed in terms of: Equal Error Rate $(E E R)$, corresponding to the error rate computed for $F R R=F A R$; $1 \%$ FAR, which is the FRR when FAR is fixed to $1 \% ; 1 \% F R R$, which is the FAR when FRR is fixed to $1 \%$.

Results in Tables 1 and 2 clearly show that the ideal selector exhibits a very small error rate. In addition, the related ROC curve reported in Figure $2 \mathrm{a}$ is below any other curve. It is worth noting that in Figure $2 \mathrm{~b}$ the ROC curve of the ideal selector is barely visible, as it lies on the FAR axis, below all the other curves. This result shows that the proposed definition of ideal selection allows attaining very small error rates. It is worth noting that the score distributions of the ideal selector reported in Figure 1 are related to results of Experiment 2.

The analysis of the results in Table 1 shows that the $D S S$ algorithm has the best single $1 \%$ FRR error, while the $D S S$ with selection threshold has the best 
single error for EER and 1\% FAR. Thus it can be concluded that the DSS with selection threshold exhibits the best performances compared to other methods used in Experiment 1. The analysis of the results in Table 2 shows that the $D S S$ algorithm exhibits the best single $1 \% F R R$ and $E E R$ values. If the $D S S$ with selection threshold is considered, while it exhibits the same value of $1 \% F R R$ as the $D S S$, it also exhibits a lower value of $1 \%$ FAR. Thus, by setting a threshold on the $D_{j}$ value, it is possible to improve the performances in terms of $1 \% F A R$.

\section{Conclusions}

In this paper the basis for score selection was presented. A definition of ideal selector was given for score-level fusion, and an algorithm implementing the concepts of the ideal selector has been proposed. The experimental results, showed that score selection is an alternative to fusion strategies. While the concepts and the techniques illustrated in the paper were used for a fingerprint multi-sensor and multi-algorithmic system, they can be used for any score-based classification system and, in particular, with any kind of multi-modal biometric systems.

\section{References}

1. J. Bigun, J. Fierrez-Aguilar, J. Ortega-Garcia and J. Gonzales-Rodriguez, Multimodal Biometric Authentication using Quality Signals in Mobile Communications, Proc. IAPR ICIAP, IEEE CS Press, pp. 2-13, Mantova, Italy, Sept. 2003

2. G. Giacinto and F. Roli, Dynamic Classifier Selection, Proc. of the First Int. Work. on MCS, 2000, Lecture Notes In Computer Science 1857, Springer, pp. 177-189

3. V. Gunes, M. Ménard, P. Loonis and S. Petit-Renaud, Combination, Cooperation and Selection of Classifiers: A State of the Art, International Journal of Pattern Recognition and Artificial Intelligence, Vol. 17, No. 8 (2003), pp. 1303-1324

4. L. Hong, A. K. Jain and S. Pankanti, Can Multibiometrics Improve Performance?, Proceedings AutoID'99, Summit, NJ, pp. 59-64

5. A.K. Jain, L. Hong and Y. Kulkarni, A Multimodal Biometric System using Fingerprint, Face and Speech, Proc. 2nd Int. Conf. on AVBPA, Mar. 1999, pp. 182-187

6. A.K. Jain, S. Prabhakar and A. Ross, Fingerprint Matching:Data Acquisition and Performance Evaluation, MSU Technical Report TR99-14, 1999

7. L.I. Kuncheva, Combining Pattern Classifiers: Methods and Algorithms, John Wiley \& Sons Inc., 2004, Chapter 6, pp. 189-202

8. G.L. Marcialis, F. Roli and P. Loddo, Fusion of Multiple Matchers for Fingerprint Verification, Proc. of the Work. on Machine Vision and Perception, Sept. 2002

9. G.L. Marcialis and F. Roli, Fingerprint Verification by Fusion of Optical and Capacitive Sensors, Pattern Recognition Letters, Vol. 25 (11), 2004, pp. 1315-1322

10. S. Prabhakar and A. K. Jain, Decision-level Fusion in Biometric Verification, Pattern Recognition, Vol. 35 (4), 2002, pp. 861-874

11. A.Ross, A. Jain and J.Z. Qian, Information fusion in Biometrics, Proc. International Conference on Image Processing, Rochester, New York, Sept. 22-25, 2002

12. K. Woods, W.P. Kegelmeyer and K. Bowyer, Combination of Multiple Classifiers Using Local Accuracy Estimates, IEEE Trans. PAMI, vol. 18 (4), 1997, pp. 405-410 